Chapter 11

\title{
The Role of Transcription Factors in Wheat Under Different Abiotic Stresses
}

\author{
Mahdi Rahaie, Gang-Ping Xue and Peer M. Schenk \\ Additional information is available at the end of the chapter \\ http://dx.doi.org/10.5772/54795
}

\section{Introduction}

Abiotic stresses such as drought, salinity and low temperature adversely affect the growth and productivity of plants. The development of stress-tolerant crops will be essential for agriculture in the many regions in the world that are prone to such stresses [48].

Wheat (Triticum aestivum L.) is one of the four major cereals in the world. As one of the most important agricultural crops, wheat is a staple food crop for a large portion of the world's population [83]. It is grown under both rain-fed and irrigated cultivation and thus under conditions subjected to many environmental stresses [68]. Unfortunately, its production is severely affected by adverse environmental stresses. Therefore, the identification and functional study of stress responsive genes will elucidate the molecular mechanisms of the plant stress response and tolerance, and will ultimately lead to improvement of stress tolerance in wheat $[58,83]$. Abiotic stresses such as drought and high salinity lead to wide range of biochemical, physiological and morphological, responses in plants in the process of adaptation to these adverse conditions. These adaptations require a large number of changes in gene expression. Many of the differentially expressed gene products protect plant cells from damage, such as dehydrins, enzymes for the synthesis of osmolytes and enzymes for the removal of reactive oxygen species (ROS) [3]. The production of these functional proteins is widely regulated by specific transcription factors [58, 65].

Transcription factors (TFs) are considered to be the most important regulators that control genes and gene clusters [49]. Many families of transcription factors have been demonstrated to play a role in stress responses in plants. Among them, the bZIP [69], WRKY [43], AP2 [64], NAC [78] and $\mathrm{C} 2 \mathrm{H} 2$ zinc finger [31] families comprise a high proportion of abiotic stressresponsive members [58]. One TF gene can control the expression of a broad range of target genes through binding to the specific cis-acting element in the promoters of these genes, also 
referred to as regulon [49]. Transcription factors (TFs) provide a possibility for plants to overcome and respond to biotic and abiotic stresses and are also involved in modulating developmental processes $[45,83]$.

Until now, several major regulons involved in response to abiotic stress have been identified in Arabidopsis. Recent studies have demonstrated that DREB1/CBF, DREB2, AREB/ABF, and NAC regulons have important functions in response to abiotic stresses in rice [49]. Significant advances have been made in recent years towards identifying regulatory genes involved in stress responses which confer abiotic stress tolerance in plants [18].

In this review, we provide an overview of the functions of different TF family members with particular emphasis on the role of bZIP, bHLH, WRKY, MYB, and NAC TFs and their involvement in abiotic stress responses in wheat.

\section{2. bZIP transcription factors}

Basic region/leucine zipper (bZIP) TFs possess a basic region that binds DNA and a leucine zipper dimerization motif. The bZIP domain comprises two structural features located on a contiguous $\alpha$-helix: a basic region of about 16 amino acid residues with a nuclear localization signal, an invariant N-x7-R/K motif to contact the DNA as well as a heptad repeat of leucines or other bulky hydrophobic amino acids located exactly nine amino acids towards the Cterminus, to create an amphipathic helix. When binding to DNA, two subunits adhere through interactions of the hydrophobic sides of their helices, which create a superimposing coiled-coil structure (zipper). The capability to form homo- and heterodimers is governed by the electrostatic attraction and repulsion of polar residues adjacent to the hydrophobic interaction surface of the helices. Proteins with bZIP domains are present in all eukaryotes analyzed to date and bZIP proteins typically bind to DNA sequences with an ACGT core. Plant bZIPs bind to the A-box (TACGTA), C-box (GACGTC) and G-box (CACGTG), but there are also reports of nonpalindromic binding sites for bZIPs [24]. Based on the sequence similarities of common domains, 75 bZIP protein members have been divided into ten subgroups in Arabidopsis [24, 35]. In plants, bZIP transcription factors present a divergent family of TFs which regulate processes including light and stress signaling, seed maturation, pathogen defense, and flower development $[24,59]$.

The plant hormone abscisic acid (ABA) plays an essential role in maturation and germination in seeds, as well as mediating adaptive responses to abiotic environmental stresses. ABA induces the expression of many genes, including late-embryogenesis-abundant (LEA) genes. HVA1 is one of the LEA genes whose expression is affected by ABA. Analysis of the interplay between ABA and TaABF1 as a bZIP factor in the aleurone cells of imbibing wheat grains by Keyser [32] indicated that the two are not additive in their induction of the HVA1 promoter. It has been shown that TaABF1 may undergo an ABA-induced posttranslational modification. However, the lack of synergism between ABA and TaABF1 overexpression in HVA1 induction does not support this conclusion. These findings indicate that the branch of ABA signaling leading to HVA1 is more complex [32]. 
Kobayashi et al. [35] isolated a wheat lip19 (encoding bZIP-type transcription factors) homologue, Wlip19 and analyzed its expression in response to cold stress. Wlip19 expression was stimulated by low temperature in seedlings and was higher in a freezing-tolerant wheat cultivar than in a freezing-sensitive variety. Wlip19 expression was also activated by drought and exogenous ABA treatment. Heterologous expression of Wlip19 in tobacco has showed a significant increase in abiotic stress tolerance, especially freezing tolerance.

It was indicated that WLIP19 acts as a transcriptional regulator of Cor/Lea genes in the development of abiotic stress tolerance by enhancement of expression of four wheat Cor/Lea genes, Wdhn13, Wrab17, Wrab18, and Wrab19, in wheat callus and tobacco plants. Furthermore, direct protein-protein interactions between WLIP19 and another bZIP-type transcription factor in wheat, the OBF1 homologue TaOBF1, was observed, implying that this interaction is conserved in cereals [35].

Expression analysis of a group of bZIP candidate genes in long term salinity into contrasting cultivars of wheat by reverse northern blot showed that $b$ ZIP1 (CN011839] was up-regulated in a susceptible variety (Chinese Spring) and down-regulated in a tolerant cultivar (Mahouti) during salt stress. Sequence analysis by BLASTX showed that this gene's protein has two homologues in Arabidopsis (AtZIP56, E value $=1 \mathrm{e}^{-2} 0$ ] and wheat $\left(\mathrm{TaABF}, \mathrm{E}\right.$ value $=6 \mathrm{e}^{-5}$ ]. The results of published work showed that $T a A B F$ mRNA accumulates together with $P K A B A 1$ mRNA (an ABA-induced protein kinase) during wheat grain maturation and dormancy acquisition and $T a A B F$ transcripts increase transiently during imbibitions of dormant grains. In contrast to $P K A B A 1 \mathrm{mRNA}, T a A B F$ transcripts are seed specific and were not markedly produced in vegetative tissues in response to ABA application or abiotic stress $[29,59]$.

HY5, another bZIP1 homologue from the H group of Arabidopsis bZIPs, is involved in photomorphogenesis regulation. The necessary TF for response to a broad spectrum of wavelengths of light acts as a positive regulator in photomorphogenesis by regulating the expression of downstream genes in response to a light signal. Interestingly, HY5 integrates both hormone and light signaling pathways. In hy 5 mutants, the expression of hundreds of genes is affected by UV-B or blue light [7]. Another affected bZIP by salt stress in tolerant genotype was bZIP5 (CV765814] from group I bZIPs. The analysis of group I genes from several species indicates that they might play a role in vascular development $[24,59]$.

\section{3. bHLH transcription factors}

Basic helix-loop-helix (bHLH) proteins comprise a group of diverse transcription factors with highly diverse functions and are present in both plants and animals. The bHLH domain, the characteristic of this family, consists of about 60 amino acids with two functionally distinct regions. The basic region at the $\mathrm{N}$-terminal end of the domain is required for DNA binding while the C-terminal HLH region functions as a dimerization domain. These TFs in plants, act as transcriptional regulators required for phytochrome signaling, anthocyanin biosynthesis, fruit dehiscence, carpel, and epidermal development, as well as for stress response. However, the biological function of most members of this gene family in plant has not yet been elucidated [37]. 
Gene expression analysis by reverse northern blot has shown that two selected candidate wheat bHLHs (bHLH2: CA599618 and bHLH3: CJ685625] are affected by salt stress in a tolerant wheat cultivar. The BLASTx results showed that both have a homologue in wheat, bHLH94 (E value $=5 \mathrm{e}^{-85}$ for $\mathrm{bHLH} 2$ and $\mathrm{E}$ value $=5 \mathrm{e}^{-102}$ for $\mathrm{bHLH} 3$. AtAIB was another homologue for bHLH3 from Arabidopsis involved in the regulation of ABA signaling in Arabidopsis and plays a role in drought tolerance and ABA treatment response [37, 59]. The high homology (E value $=2 \mathrm{e}^{-5} 1$ ] between these orthologues and the result of reverse northern blot hybridizations in that research indicate that these two bHLH gene may have an important function in tolerance to salt stress in wheat [59].

\section{WRKY transcription factors}

WRKY transcription factors have been studied in plants extensively in the last two decades. First Ishiguro and Nakamura [23] identified a WRKY protein in sweet potato (Ipomoea batatas); since then many other members of this TF family have been cloned and functionally characterized in plants, including wild oats (Avena fatua) [62], parsley (Petroselinum crispum) [63], tobacco (Nicotiana tabacum) [8, 19, 34, 61], Arabidopsis thaliana [12], potato (Solanum tuberosum) [5, 14], orchardgrass (Dactylis glomerata) [2], winter bittersweet nightshade (Solanum dulcamara) [22], desert legume (Retama raetam) [55], barley (Hordeum vulgare) [66], rice (Oryza sativa) [41], cotton (Gossypium arboreum) [77], and coconut (Cocos nucifera) [44]. More recently, WRKY family TFs were also identified in lower plants including ferns (Ceratopteris richardii), mosses (Physcomitrella patens) [4], a smile mode (Dictyostelium discoideum) and the protist (Giardia lamblia) [81, 73].

A WRKY domain of about 60 amino acids is a characteristic of WRKY proteins. This domain comprises the absolutely conserved sequence WRKYGQK followed by a zinc finger motif. The WRKY domain binds to the $\mathrm{W}$ box ([T][T]TGAC[C/T]) of target gene promoters to modulate transcription [10, 73]. It should be mentioned that in spite of the strong conservation of their DNA-binding domain, the overall structures of WRKY TFs are highly divergent. WRKY TF family members are grouped into three distinct groups based on the number and type of the WRKY domains which might also reflect their different functions [59]. WRKY TFs with two WRKY domains belong to group I and members of group II and group III possess one WRKY domain. Group I and group II have a $\mathrm{C} 2 \mathrm{H} 2$ zinc finger motif, while in group III, the WRKY domain contains a C2HC motif. WRKY TFs can then be further classified into different subgroups based on their phylogenetic clades. The WRKY family is one of the TF families for which the regulatory role in biotic and abiotic stresses has been demonstrated in plants. These include infection of bacteria, fungi, oomycetes and viruses, treatment with salicylic acid (SA) or $\mathrm{H}_{2} \mathrm{O}_{2}$, mechanical stimulation, drought, cold, wounding, high-salinity and UV radiation.

Most WRKY TFs of group III play a role in plant defense signaling pathways. Some members of the WRKY family may have key functions in plant development, such as embryo development, fruit maturation, tannin synthesis in the seed coat, maturation of root cells, morphogenesis of trichomes, senescence, and dormancy. Furthermore, some of WRKY family 
members have a role in hormone signaling such as OsWRKY71 and OsWRKY51 which were ABA-inducible and could repress GA signaling transduction in aleurone cells.

Wu et al. [73] obtained sequences for 15 wheat cDNAs encoding putative WRKY proteins. Phylogenetic analysis showed that the 15 WRKY genes classified to three major WRKY groups and expression analysis revealed that most genes were highly expressed in leaves. A few of them such as TaWRKY10 are expressed in the crown intensively and several genes are strongly up-regulated during the senescence of leaves. Eight isolated genes were responsive to high or low temperature, $\mathrm{NaCl}$ or PEG (polyethylene glycol) treatment. In addition, differential expression was also measured between wheat hybrids and its parents, and some genes were more responsive to PEG treatment in the hybrid. The authors concluded that the differential expression of these WRKY genes in the hybrid might contribute to heterosis by improving the stress tolerance in hybrids [73].

Orthologous genes are subjected to similar transcriptional regulation by orthologous TFs, suggesting that the terminal stages of signal transduction pathways leading to defense are conserved, implying a fundamental role of pathogenesis-related genes, such as PR4 genes in plant defense. This suggests that diversification between monocot and dicot plants has most likely occurred after the differentiation of WRKY functions. Proietti et al. [56] reported the ability of TaWRKY78 to bind to a W-box-containing region of the wPR4e promoter. Transient expression assays of TaWRKY78 and AtWRKY20 showed that both TFs are able to recognize the cognate cis-acting elements present in the wPR4e and AtHEL promoters [56].

Expression analysis by reverse northern blot hybridizations of a group of putative wheat WRKYs showed that WRKY1 (CN009320] and WRKY2 (CJ873146] were up-regulated in a stress-tolerant genotype. AtWRKY75 (E value $=3 \mathrm{e}^{-4} 2$ ] is a homologue from Arabidopsis for WRKY1 which is up-regulated in response to phosphorous deficit stress [15, 17, 59]. This gene also acts as positive regulator in defense responses to pathogens. Functional characterization of the WRKY2 homologue in Arabidopsis, AtWRKY33 (E value $\left.=4 \mathrm{e}^{-1} 8\right]$, showed that its expression in response to salt, mannitol (simulated drought) treatment and cold stress in shoots and roots increased but this gene was down-regulated during heat stress. It also appears that its expression is independent of SOS signaling and only partly dependent on ABA signaling, but forms part of plant responses to microbial infections [27, 40, 59].

\section{MYB transcription factors}

MYB TFs form one of the largest transcription factor families in plants. More than 200 MYB proteins are encoded in genomes of Arabidopsis and rice. MYB TFs contain one to four imperfect repeats [50-53 amino acids) in their DNA-binding domain (MYB domain) near to the Nterminus and are classified into four subfamilies [58, 83].

According to the number of repeat(s) in the MYB domain: 4R-MYB has four repeats, 3R-MYB (R1R2R3-MYB) has three consecutive repeats, R2R3-MYB has two repeats, and the MYBrelated type usually, but not always, has a single repeat [16, 28, 61]. Typically, the MYB repeat 
is 50-53 amino acids in length and contains three regularly-distributed tryptophan (or phenylalanine) residues, which can together form a hydrophobic core. Each MYB repeat forms three $\alpha$-helices: the two that are located at the C-terminus adopt a variation of the helix-turnhelix $(\mathrm{HLH})$ conformation that recognizes and binds to the DNA major groove at the specific recognition site such as C/TAACG/TG [51, 52].

Since the first plant MYB gene, C1, was isolated in Zea mays [54], research concerning different aspects of the MYB gene family, including gene number, sequence characterization, evolution, and potential functions, has been widely conducted in plants $[9,16,72]$. So far, large numbers of MYB genes have been identified in different plant species, comprising 204 members in Arabidopsis, 218 members in rice, 279 members in grapevine, 197 members in poplar, and 180 members in Brachypodium [9, 70, 72].

MYB proteins are involved in many significant physiological and biochemical processes, including the regulation of primary and secondary metabolism, the control of cell development and the cell cycle, the participation in defense and response to various biotic and abiotic stresses, and hormone synthesis and signal transduction [16, 83].

Extensive studies of the MYB gene family in various plant species have provided a better understanding of this gene family; however, little is known about this gene family in bread wheat [83].

We previously analyzed the expression levels of ten MYB TF genes from wheat (Triticum aestivum) in two recombinant inbred lines contrasting in their salt tolerance in response to salt or drought stress via quantitative RT-PCR [58]. A potential new MYB gene (TaMYBsdu1] was significantly up-regulated in leaves and roots of wheat plants subjected to long-term drought stress. Furthermore, TaMYBsdu1 showed higher transcript abundance in the salt-tolerant genotype than in the susceptible genotype under salt stress. These data suggested that TaMYBsdu1 is a potentially important regulator for wheat adaptation to both salt and drought stresses [58].

In other work, two putative MYB genes, MYB2 (DQ353858.1] and MYB3 (CJ920766] were upregulated in a tolerant variety (Mahouti) under salt stress conditions but down-regulated in the susceptible cultivar (Chinese Spring), MYB2. Sequence analysis with the BLASTx and Plant Gene Ontology assignment showed that MYB2 is a part of TaMYB1 (E value $=6 \mathrm{e}^{-15} 5$ ]. The results of a study by Lee et al. [36] show that TaMYB1 is involved in abiotic stresses responses in wheat. The expression of this gene increases during oxygen deficiency (flooding), PEG treatment (drought) and salt increases, especially in roots. In addition, its transcript gradually increases in starting ABA and PEG treatments [36]. In research conducted by Mott and Wang [46] on comparative transcriptome analysis of salt-tolerant wheat germplasm lines using wheat genome arrays, it was found that TaMYB1 was one of the up-regulated genes with 34 times higher expression levels under stress condition relative to the control. Functional analysis of the MYB2 homologue in Arabidopsis, AtMYB44 (E value=1e-59], showed that this gene was upregulated in response to drought, salt, cold and ABA treatments, especially in stomata guard cells and vascular tissue. Transgenic plants overexpressing this gene showed more tolerance to mentioned stresses compared to wide-type plants [28]. Homology analysis of MYB3 (a 
member of R2R3MYB) has shown that there is a high homology between this gene and AtMYB59 in Arabidopsis (E value $=4 \mathrm{e}^{-6} 0$ ]. It has been shown that $A t M Y B 59$ expression increases in response to phytohormones including jasmonic acid, SA, gibberellic acid and ethylene, especially in leaf and stem tissues $[38,39,59]$. But its expression level in roots and inflorescences was lower than in other organs, showing its role in hormonal signal pathways in response to biotic stresses and plant defense against pathogen attacks [38, 39, 59].

Full-length cDNA is an important resource for isolating the functional genes in wheat. Recently, Zhang et al. [83] analyzed a group of MYB genes that respond to one or more stress treatments. They isolated 60 full-length cDNA sequences encoding wheat MYB proteins. A phylogenetic tree with wheat, rice, and Arabidopsis MYB proteins was constructed to examine their evolutionary relationships and the putative functions of wheat MYB proteins based on Arabidopsis MYB proteins with known functions. Tissue-specific analysis and abiotic stress response expression profiles were carried out to find potential genes that participate in the stress signal transduction pathway, including the analysis of transgenic Arabidopsis plants expressing the MYB gene, TaMYB32 [83].

Recently, Qin et al. [56] identified a new R2R3-type MYB transcription factor gene, TaMYB33, from wheat (T. aestivum). This gene was induced by $\mathrm{ABA}, \mathrm{NaCl}$, and PEG treatments, and its promoter sequence contains the putative ABRE, MYB and other abiotic stress-related ciselements. Ectopic over-expression of this gene in Arabidopsis significantly enhanced its tolerance to drought and $\mathrm{NaCl}$ treatments, but not to $\mathrm{LiCl}$ and $\mathrm{KCl}$ stresses. The expression of two genes, AtP5CS (involved in proline synthesis) and AtZAT12 (a C2H2 zinc finger transcription factor that is involved in regulating ascorbate peroxidase expression), was induced in the TaMYB33-expressing transgenic Arabidopsis lines. This suggests that TaMYB33 promotes the ability for ROS scavenging and osmotic pressure balance reconstruction. TaMYB33 overexpression lines displayed up-regulation of $A t A A O 3$ along with down-regulation of $A t A B F 3$ and $A t A B I 1$, indicating that ABA synthesis was elevated while its signaling was constrained. The authors concluded that TaMYB33 enhances salt and drought tolerance partially via an improved ability for ROS detoxification and osmotic balance reconstruction [57].

TaMYB56 (on chromosomes 3B and 3D) in wheat was identified as a cold stress-related gene by Zhang et al. [82]. The expression levels of TaMYB56-B and TaMYB56-D were strongly induced by cold stress, but slightly induced by salt stress in wheat. Detailed characterization of the Arabidopsis transgenic plants that overexpressed TaMYB56-B revealed that TaMYB56-B is possibly involved in the responses of plants to freezing and salt stresses. The expression of some cold stress-responsive genes, such as DREB1A/CBF3 and COR15a, were found to be elevated in the TaMYB56-B-overexpressing Arabidopsis plants compared to wild-type [82].

TaMYB3R1 is another MYB gene which has been shown to be potentially involved in wheat response to drought, salt and cold stress. Cai et al. [6] cloned TaMYB3R1 from wheat (T. aestivum). TaMYB3R1 amino acid sequence shares high identity to other plant MYB3R proteins. Subcellular localization experiments in onion epidermal cells proved that TaMYB3R1 was present in the nucleus. Trans-activation assays in yeast cells confirmed that TaMYB3R1 was a TF that required the C-terminal region to activate the expression of reporter gene. DNAbinding tests showed the MSA cis-element-binding activity of TaMYB3R1. TaMYB3R1 
expression was induced following ABA treatment and gradually increased expression until $72 \mathrm{~h}$ after salt or cold treatment. In contrast, PEG treatment lead to an early expression peak at $6 \mathrm{~h}$ after treatment, and then gradually decreased [6].

Zhang et al. [84] identified TaMYB32 as a salt stress-related gene, during the bulk sequencing of full length cDNAs in wheat (T. aestivum). The sequences of TaMYB32 were cloned from different varieties of hexaploid wheat and its diploid ancestors. Sequence analysis indicated that two types of sequences existed in the diploid ancestors and four in the hexaploid wheat. One of the sequences was identical in both diploid and hexaploid wheat. This implied that TaMYB32 was conserved during the evolution of wheat. The genomic TaMYB32 sequences proved to be non-intron genes after comparing with their cDNA sequences. TaMYB32 was mapped onto the homoeologous group 6 of wheat using the electronic mapping strategy, and two copies of the gene were found in each genome of hexaploid wheat. Homologous analysis found that TaMYB32 had a similarity with some R2R3-MYB proteins from rice (Oryza sativa L.) and maize (Zea mays L.) as high as $72.4 \%$ and $73.7 \%$, respectively. The expression of TaMYB32 in roots, stems, leafs, pistils, and anthers in wheat, was induced by salt stress [84].

\section{NAC transcription factors}

The first sequenced cDNA encoding a NAC protein was the RESPONSIVE TO DEHYDRATION 26 (RD26] gene in Arabidopsis [80]. The NAC domain was characterized based on consensus sequences from Petunia NAM and Arabidopsis ATAF1/2 and CUC2 proteins [1]. Many NAC TFs, including Arabidopsis CUC2, play important roles in plant development. Some NAC genes mediate viral resistance [48], while others are up-regulated during wounding and bacterial infection [11]

NAC domains mediate transcriptional regulation of various biological processes by forming a helix-turn-helix structure that specifically binds to the target DNA [1]. NAC TFs are quite diverse in their C-terminal sequences which possess either activation or repression activity. More than 100 NAC genes have so far been identified in Arabidopsis and rice which can be categorized into six major groups. Phylogenetic analyses suggest that these were already present in an ancient moss lineage. NAC TFs play a range of important roles during plant development and abiotic stress responses [48]. Many plant growth and developmental processes are regulated by NAC TFs, including shoot apical meristem formation, lateral root development, senescence, cell wall development, and secondary metabolism. A large number of NAC TFs are also differentially expressed in responses to abiotic and biotic stresses [74] and transgenic Arabidopsis and rice plants overexpressing stress-responsive NAC genes have displayed improved drought tolerance. These studies indicate that stress-responsive NAC transcription factors have important roles for the control of abiotic stress tolerance and that their overexpression can improve stress tolerance via biotechnological approaches [48].

Interestingly, rice plants overexpressing OsNAC6 possessed enhanced tolerance to abiotic (dehydration, high salinity) as well as biotic stresses (blast disease) [47]. The Arabidopsis NAC TF, ATAF2, is induced by salicylic acid (SA) and methyl jasmonate (MeJA) treatments, and is 
also differentially expressed following wound stress response [22]. The potato StNAC gene shows induced expression in responses to Phytophthora infestans infection and wounding treatment [23]. Barley plants with the HvNAC6 gene knocked down show penetration resistance in epidermal cells when inoculated with virulent isolates of Blumeria graminis $\mathrm{f}$. sp. hordei [25]. Overexpression of rice OsNAC4 resulted in hypersensitive response (HR) cell death; and in the OsNAC4 knocked down lines, HR cell death was markedly decreased in response to the avirulent bacterial strain (Acidovorax avenae N1141] [67]. Therefore, it seems that plant NAC TFs play multiple roles in defense responses to pathogen attack as well as exogenous stimuli [74].

Although these transcription factors can bind to the same core NAC recognition sequence, recent reports have shown that the different NAC TFs have different functions in plant development. In addition, NAC proteins can form homo- or hetero-dimers. Stress-responsive NAC TFs can be used for improving stress tolerance in transgenic plants, although the mode of action appears complex in plants. Recent reports support the notion of substantial crosstalk between plant growth and stress responses. In rice, Kikuchi et al. [33] characterized the molecular properties of eight NAC genes (OsNAC1 to OsNAC8].

In contrast to Arabidopsis, the NAC regulon may have additional roles in monocot plants. Important future tasks will, therefore, lie in the comparative analysis of gene expression patterns and the identification of their target genes to determine the function of these genes in plant development and tolerance to abiotic and biotic stresses [49].

Xia et al. [74] reported the full-length cDNA sequence of a novel wheat (T. aestivum) NAC TF, TaNAC8, (using in silico cloning, reverse transcription PCR and 3 ' rapid amplification of cDNA ends PCR methods. TaNAC8 shows strong homology to rice OsNAC8 with an N-terminal NAC domain and a trans-membrane helices motif in the C-terminus. Yeast one hybrid assays confirmed that TaNAC8's C-terminal region acted as transcriptional activator. Inoculation of wheat with an incompatible isolate of the stripe rust pathogen Puccinia striiformis $\mathrm{f}$. sp. tritici or treatments with MeJA or ethylene led to increased TaNAC8 transcription in leaves $24 \mathrm{~h}$ post inoculation/treatment. However, SA and ABA had no significant effect on gene expression. Abiotic stress treatments, including high salinity, PEG and low-temperature, also induced TaNAC8 expression, suggesting that TaNAC8 may function as a transcriptional activator involved in wheat defense responses to both abiotic and biotic stresses [74].

Mao et al. [42] obtained a fragment of TaNAC2 from suppression subtractive cDNA libraries of wheat treated with PEG, and its full-length cDNA was obtained by screening a full-length wheat cDNA library. Gene expression profiling indicated that TaNAC2 was involved in response to drought, salt, cold, and ABA treatment. Overexpression of TaNAC2 in Arabidopsis resulted in enhanced tolerances to drought, salt, and freezing stresses which coincided with enhanced expression of abiotic stress-response genes and several physiological indices [42].

TaNAC4 encodes another NAC TF in wheat high homology with rice OsNAC4 [73]. Functional analysis using onion epidemical cells and yeast one-hybrid assays confirmed that TaNAC4 functions as a transcriptional activator. TaNAC4 expression was induced in wheat leaves by infection with stripe rust, and also by MeJA, ABA and ethylene treatments. However, SA had no 
obvious effect on TaNAC4 expression. Similar to TaNAC8, abiotic stresses such as high salinity, wounding, and low-temperature also induced TaNAC4 expression, suggesting a role of TaNAC4 as a transcriptional activator during biotic and abiotic stresses responses in wheat [75].

Rahaie et al. [59] have shown that NAC67 (BU672229], a putative member of the NAC family was up-regulated during salt stress treatment. The encoded protein has a close homologue in wheat (TaNAC69, E value $\left.=2 \mathrm{e}^{-15} 1\right][59,78]$. Xue et al. [78] demonstrated the role of TaNAC69 in response to abiotic stresses including drought, cold and ABA treatments. Expression analysis of three highly homologous TaNAC69 genes showed that these were up-regulated during the above-mentioned stresses, especially drought stress. Besides their up-regulation by drought, TaNAC69 genes were expressed at high levels in the root under unstressed conditions. This suggests that TaNAC69 genes are not just involved in drought stress, but may also be required in normal cellular activities of roots [78]. Over-expression of TaNAC69 in transgenic wheat leads to enhanced dehydration tolerance and improvement of water use efficiency [79]. AtNAC2 is also a NAC67 homologue in Arabidopsis which is involved in salinity stress, ABA, ACC and NAA treatment in Arabidopsis, but AtNAC2 induction by salt stress requires the ethylene and auxin signaling pathways. It has been shown that the expression level of AtNAC2 in roots and flowers has been higher than in other tested tissues [20,59].

\section{Enhanced abiotic stress resistance by genetic manipulation of a transcription factor linked to crop yield improvement in the field}

In the past decade numerous transgenic plant studies have demonstrated that the improvement of abiotic stress resistance can be achieved by genetic manipulation of transcription factors. However, many resistant transgenic lines with constitutive over-expression of a transcription factor exhibit a slower rate of growth under non-stress conditions. Field trials have also shown that some transgenes tend to have a negative effect on grain yield under normal growth conditions [76]. This phenomenon can theoretically result from the following two causes: (i) genes that are induced during stress generally have a negative impact on the growth and yield, and (ii) the energetic cost of the stress-related metabolite accumulation due to over-expression of a transcription factor. Therefore, the expression of a transcription factor needs to be tailored to meet the requirement for plant stress adaptation if the crop yield is concerned. Any reduction of crop yield under normal growth conditions could potentially override a marked yield advantage under stress.

The expression of a transcription factor can be tailored to stress adaptation by using a stressinducible promoter. For example, transgenic Arabidopsis plants carrying a drought inducible promoter-driven $D R E B 2 A$ gene exhibit the improved drought resistance with no significant difference in growth rate under normal growth conditions [64]. Other aspects for consideration of minimizing the negative impact of transgene expression on growth and yield include the appropriate expression level of the transgene and cell specificity. Recently, a root-specific promoter has been used for driving expression of drought-upregulated transcription factors for engineering drought tolerance [26, 60]. Most interestingly, a number of transcription factors 
have been shown to improve crop yield under field conditions when they are over-expressed in transgenic plants (Table 1). These studies clearly demonstrate that genetic manipulation of stress-responsive transcription factors has potential for improvement of crop yield in the future, including wheat.

\begin{tabular}{|c|c|c|c|c|}
\hline Gene description & Host & Expression mode & Acquired traits & Reference \\
\hline Rice SNAC1 (NAC) & Rice & Constitutive OE & $\begin{array}{l}\text { Improved spikelet fertility under } \\
\text { drought and reduced transpiration }\end{array}$ & 21 \\
\hline Maize NF-YB2 (NF-YB) & Maize & Constitutive $\mathrm{OE}$ & $\begin{array}{l}\text { Less wilting, delayed senescence, higher } \\
\text { photosynthesis rate and improved yield } \\
\text { under drought }\end{array}$ & \\
\hline ZAT10 ( $\mathrm{C}_{2} \mathrm{H}_{2}$ zinc finger $)$ & rice & $\begin{array}{l}\text { Drought-inducible or } \\
\text { constitutive OE }\end{array}$ & $\begin{array}{l}\text { Improved spikelet fertility and grain } \\
\text { yield per plant under drought }\end{array}$ & 76 \\
\hline CBF3 (AP2) & rice & Drought-inducible OE & $\begin{array}{l}\text { Improved spikelet fertility and grain } \\
\text { yield per plant under drought }\end{array}$ & 76 \\
\hline Rice AP37 (AP2) & Rice & Constitutive OE & $\begin{array}{l}\text { Enhanced drought resistance and grain } \\
\text { yield under severe drought conditions }\end{array}$ & 53 \\
\hline Rice NAC10 (AP2) & Rice & Root-specific OE & $\begin{array}{l}\text { Enhanced drought resistance and grain } \\
\text { yield under both normal and drought } \\
\text { conditions }\end{array}$ & 26 \\
\hline Rice NAC9 (AP2) & Rice & Root-specific OE & $\begin{array}{l}\text { Enhanced drought resistance and grain } \\
\text { yield under both normal and drought } \\
\text { conditions }\end{array}$ & 60 \\
\hline
\end{tabular}

Table 1. Transgenic crops with over-expression of a transcription factor improve yield under field conditions

\section{Conclusion and prospective}

Abiotic stresses such as drought, salinity and low temperature adversely affect the growth and productivity of plants. Successful breeding of stress-tolerant varieties will be vital to ensure food supply in areas that are prone to such stresses. Recent advances towards identifying potential abiotic stress tolerance genes have been made. Many TFs and other regulatory genes involved in stress responses have been identified, giving rise to the idea that plants have developed flexible molecular and cellular response mechanisms to respond to various abiotic stresses. bZIP, WRKY, bHLH, MYB and NAC transcription factors represent the major groups of regulatory genes of which some members are found to be involved in abiotic stress responses in plants. To date, the functions of a number of abiotic stress-responsive transcription factor genes have been studied in many different species, including wheat. Recent studies have 
indicated that certain stress-induced TF genes play significant roles in wheat stress tolerance. These studies enhance our understanding of the mechanisms of responses and tolerance to abiotic stress in wheat. Also, it provides us a collection of suitable candidate genes for overor under-expression studies in transgenic wheat aiming to achieve increased abiotic stress tolerance.

In the future, a systems biology approach using reverse genetics, functional genomics and proteomics, as well as metabonomics during various developmental stages and stress conditions will provide us with critical information to elucidate the function of the different stressresponsive TFs and their relationship in transcriptional control in wheat.

In the years ahead, the verification of abiotic stress tolerance and agronomic traits of transgenic wheat utilizing stress-responsive TF genes should be evaluated under harsh field conditions over several years. It can be expected that with increases in climatic variations, more robust cultivars that withstand a wide variety of stresses will be superior over those that are high yielding under optimal conditions. To this end, it will be necessary to clarify the differential function of the individual stress-responsive TF genes from different families of TFs for the control of abiotic stress tolerance and other biological processes including biotic stress tolerance, growth regulation, senescence and yield in order to fully utilize the potential of transcription factors.

\section{Author details}

Mahdi Rahaie ${ }^{1}$, Gang-Ping Xue ${ }^{2}$ and Peer M. Schenk ${ }^{3 *}$

1 Department of Life Science Engineering, Faculty of New Science and Technology, University of Tehran, Tehran, Iran

2 Commonwealth Scientific and Industrial Research Organization Plant Industry, Queensland Bioscience Precinct, St. Lucia, Queensland, Australia

3 School of Agriculture and Food Sciences, The University of Queensland, St. Lucia, Queensland, Australia

\section{References}

[1] Aida, M, Ishida, T, Fukaki, H, Fujisawa, H, \& Tasaka, M. (1997). Genes involved in organ separation in Arabidopsis: an analysis of the cup-shaped cotyledon mutant. Plant Cell, , 9, 841-857.

[2] Alexandrova, K. S, \& Conger, B. V. (2002). Isolation of two somatic embryogenesisrelated genes from orchardgrass (Dactylis glomerata). Plant Sci., , 162, 301-7. 
[3] Bartels, D, \& Sunkar, R. (2005). Drought and salt tolerance in plants. Crit. Rev. Plant Sci., , 24, 23-58.

[4] Bekir, U, \& Somssich, I. E. (2004). WRKY transcription factors: from DNA binding towards biological function. Curr. Opin. Plant Biol., , 7, 491-8.

[5] Beyer, K, Binder, A, Boller, T, et al. (2001). Identification of potato genes induced during colonization by Phytophthora infestans. Mol Plant Pathol., , 2, 125-34.

[6] Cai, H, Tian, S, Liu, C, \& Dong, H. (2011). Identification of a MYB3R gene involved in drought, salt and cold stress in wheat (Triticum aestivum L.). Gene, , 485, 146-152.

[7] Chang, C, Li, S. J, Chen, Y. -H, Chen, L. -T, Hsieh, W. -C, Shin, W. -P, Jane, J, Chou, W. -N, Choi, S. -J, Hu, G, Somerville, J-M, Wu, S, \& Lzf, S-H. a HY5-regulated transcriptional factor, functions in Arabidopsis de-etiolation. Plant J., , 54, 205-219.

[8] Chen, C. H, \& Chen, Z. X. (2000). Isolation and characterization of two pathogenand salicylic acid-induced genes encoding WRKY DNA binding proteins from tobacco. Plant Mol Biol., , 42, 387-96.

[9] Chen, Y. H, Yang, X. Y, He, K, et al. (2006). The MYB transcription factor superfamily of Arabidopsis: expression analysis and phylogenetic comparison with the rice MYB family. Plant Molecular Biology, , 60, 107-124.

[10] Chen, L, Song, Y, Li, S, Zhang, L, Zou, C, \& Yu, D. (2011). The role of WRKY transcription factors in plant abiotic stresses. Biochimica et Biophysica Acta,

[11] Collinge, M, \& Boller, T. (2001). Differential induction of two potato genes, Stprx2 and StNAC, in response to infection by Phytophthora infestans and to wounding. Plant Mol Biol., 46(5):521e9.

[12] De Pater, S, Greco, V, Pham, K, et al. (1996). Characterization of a zinc dependent transcriptional activator from Arabidopsis. Nucleic Acids Res., , 24, 4624-31.

[13] Delessert, C, Kazan, K, Wilson, I. W, Van Der Straeten, D, \& Manners, J. Dennis Elizabeth S, et al. (2005). The transcription factor ATAF2 represses the expression of pathogenesis-related genes in Arabidopsis. Plant J., 43:745e57.

[14] Dellagi, A, Heilbronn, J, Avrova, A. O, et al. (2000). A potato gene encoding a WRKYlike transcription factor is induced in interactions with Erwinia carotovora subsp. atroseptica and Phytophthora infestans and is coregulated with class I endochitinase expression. Mol. Plant Microbe Interact., , 13, 1092-101.

[15] Devaiah, B. N, Karthikeyan, A. S, \& Raghothama, K. G. (2007). WRKY75 Transcription Factor Is a Modulator of Phosphate Acquisition and Root Development in Arabidopsis. Plant Physiol., , 143, 1789-1801.

[16] Dubos, C, Stracke, R, Grotewold, E, Weisshaar, B, Martin, C, \& Lepiniec, L. (2010). MYB transcription factors in Arabidopsis. Trends Plant Sci., , 15, 573-581.

[17] Encinas-villarejo, S, Maldonado, A. M, Amil-ruiz, F, Santos, B. d. 1, Romero, F, Pliegoalfaro, F, Oz-blanco, J. M, \& Caballero, J. L. (2009). Evidence for a positive regulatory 
role of strawberry (Fragaria ananassa) Fa WRKY1 and Arabidopsis AtWRKY75 proteins in resistance. J. Exp. Bot., , 60(11), 3043-3065.

[18] Galiba, G, Vagujfalvi, A, Li, C, Solte, A, \& Sz, J. Dubcovsky. (2009). Regulatory genes involved in the determination of frost tolerance in temperate cereals, Plant Sci., , 176, 12-19.

[19] Hara, K, Yagi, M, Kusano, T, et al. (2000). Rapid systemic accumulation of transcripts encoding a tobacco WRKY transcription factor upon wounding. Mol. Gen. Genet., , 263, 30-7.

[20] He, X-J, Mu, R-L, Cao, W-H, Zhang, Z-G, Zhang, J-S, \& Chen, S-Y. (2005). AtNAC2, a transcription factor downstream of ethylene and auxin signaling pathways, is involved in salt stress response and lateral root development. Plant J., , 44, 903-916.

[21] Hu, H, Dai, M, Yao, J, et al. (2006). Overexpressing a NAM, ATAF, and CUC (NAC) transcription factor enhances drought resistance and salt tolerance in rice. Proc. Natl. Acad. Sci. U.S.A., , 103, 12987-12992.

[22] Huang, T, \& Duman, J. G. (2002). Cloning and characterization of a thermal hysteresis (antifreeze) protein with DNA-binding activity from winter bittersweet nightshade, Solanum dulcamara. Plant Mol Biol., , 48, 339-50.

[23] Ishiguro, S, \& Nakamura, K. (1994). Characterization of a cDNA encoding a novel DNAbinding protein, SPF1, that recognizes SP8 sequences in the 5, upstream regions of genes coding for sporamin and b-amylase from sweet potato. Mol Gen Genet., , 244, 563-71.

[24] Jakoby, M, Weisshaar, B, Dröge-laser, W, Vicente-carbajosa, J, Tiedemann, J, Kroj, T, \& Parcy, F. (2002). bZIP transcription factors in Arabidopsis. Trends in Plant Sci., , 7, 106-111.

[25] Jensen, M. K, Rung, J. H, Gregersen, P. L, Gjetting, T, Fuglsang, A. T, Hansen, M, et al. (2007). The HvNAC6 transcription factor: a positive regulator of penetration resistance in barley and Arabidopsis. Plant Mol Biol., 65:137e50.

[26] Jeong, J. S, Kim, Y. S, Baek, K. H, Jung, H, Ha, S, Choi, H, Kim, Y. D, Reuzeau, M, Kim, C, \& Root-specific, J. -K. expression of OsNAC10 improves drought tolerance and grain yield in rice under field drought conditions. Plant Physiol., , 153, 185-197.

[27] Jiang, Y, \& Deyholos, M. K. (2009). Functional characterization of Arabidopsis NaClinducible WRKY25 and WRKY33 transcription factors in abiotic stresses. Plant Mol. Biol., , 69, 91-105.

[28] Jin, H, \& Martin, C. (1999). Multifunctionality and diversity within the plant MYB-gene family. Plant Mol. Biol., , 41, 577-585.

[29] Johnson, R. R, Wagner, R. L, Verhey, S. D, \& Walker-simmons, M. K. (2002). The abscisic acid-responsive kinase PKABA1 interacts with a seed-specific abscisic acid response 
element-binding factor, TaABF, and phosphorylates TaABF peptide sequences. Plant Physiol., , 130, 837-846.

[30] Jung, C, Seo, J. S, Han, S. W, Koo, Y. J, Kim, C. H, Song, S. I, Nahm, B. H, Choi, Y. D, \& Cheong, J. J. (2008). Overexpression of AtMYB44 enhances stomatal closure to confer abiotic stress tolerance in transgenic Arabidopsis. Plant Physiol., , 146, 623-635.

[31] Kam, J, Gresshoff, P. M, Shorter, R, \& Xue, G. P. H2 zinc finger subfamily of transcription factors in Triticum aestivum is predominantly expressed in roots and enriched with members containing an EAR repressor motif and responsive to drought stress. Plant Mol Biol., , 67, 305-322.

[32] Keyser, B. R. (2010). The wheat bZIP factor, TaABF1, mediates ABA-induced gene expression in bombarded barley aleurone layers. Honors Theses. Paper 593. http:// digitalcommons.colby.edu/honorstheses/593.

[33] Kikuchi, K, Ueguchi-tanaka, M, Yoshida, K. T, Nagato, Y, Matsusoka, M, \& Hirano, H. Y. (2000). Molecular analysis of the NAC gene family in rice, Mol. Gen. Genet., , 262, 1047-1051.

[34] Kim, C. Y, \& Zhang, S. (2004). Activation of a nitrogen-activated protein kinase cascade induces WRKY family of transcription factors and defense genes in tobacco. Plant J., , $38,142-51$.

[35] Kobayashi, F, Maeta, E, Terashima, A, Kawaura, K, \& Ogihara, Y. and Shigeo Takumi. (2008). Development of abiotic stress tolerance via bZIP-type transcription factor LIP19 in common wheat. J. of Exp. Botany, , 59, 891-905.

[36] Lee, T. G, Jang, C. S, Kim, J. Y, Kim, D. S, Park, J. H, Kim, D. Y, \& Seo, Y. W. (2007). A Myb transcription factor (TaMyb1) from wheat roots is expressed during hypoxia: roles in response to the oxygen concentration in root environment and abiotic stresses. Physiol. Plant., , 129, 375-385.

[37] Li, H, Sun, J, Xu, Y, Jiang, H, Wu, X, \& Li, C. (2007). The bHLH-type transcription factor AtAIB positively regulates ABA response in Arabidopsis. Plant Mol Biol., , 65, 655-665.

[38] Li, J, Li, X, Guo, L, Lu, F, Feng, X, He, K, Wei, L, Chen, Z, Qu, L-J, \& Gu, H. (2006). A subgroup of MYB transcription factor genes undergoes highly conserved alternative splicing in Arabidopsis and rice. J Exp Bot., , 57, 1263-1273.

[39] Libault, M, Wan, J, Czechowski, T, Udvardi, M, \& Stacey, G. (2007). Identification of 118 Arabidopsis Transcription Factor and 30 Ubiquitin-Ligase Genes Responding to Chitin, a Plant-Defense Elicitor. Mol. Plant-Microb. Interact., , 20, 900-911.

[40] Lippok, B, Birkenbihl, R. P, Rivory, G, Brümmer, J, Schmelzer, E, Logemann, E, \& Somssich, I. E. (2007). Expression of AtWRKY33 Encoding a Pathogenor PAMPResponsive WRKY Transcription Factor Is Regulated by a Composite DNA Motif Containing W Box Elements. Mol. Plant-Microb. Interact., , 20(4), 420-429. 
[41] Liu, X. Q, Bai, X. Q, Qian, Q, et al. (2005). OsWRKY03, a rice transcriptional activator that functions in defense signaling pathway upstream of OsNPR1. Cell Res., , 15, 593-603.

[42] Mao, X, Zhang, H, Qian, X, Li, A, Zhao, G, \& Jing, R. (2012). TaNAC2, a NAC-type wheat transcription factor conferring enhanced multiple abiotic stress tolerances in Arabidopsis. J. Exp. Botany, doi:10.1093/jxb/err462. , 1-14.

[43] Mare, C, Mazzucotelli, E, Crosatti, C, Francia, E, Stanca, A. M, \& Cattivelli, L. (2004). Hv-WRKY38: a new transcription factor involved in cold and drought-response in barley. Plant Mol Biol , 55, 399-416.

[44] Mauro-herrera, M, Meerow, A. W, Borrone, J, et al. (2006). Ten informative markers developed from WRKY sequences in coconut (Cocos nucifera). Mol Ecol., , 6, 904-6.

[45] Mitsuda, N, \& Ohme-takagi, M. (2009). Functional analysis of transcription factors in Arabidopsis. Plant Cell Physiol., , 50, 1232-1248.

[46] Mott, I. W, \& Wang, R. R. C. (2007). Comparative transcriptome analysis of salt-tolerant wheat germplasm lines using wheat genome arrays. Plant Sci., , 173, 327-339.

[47] Nakashima, K, Tran, L. P, Nguyen, D. V, Fujita, M, Maruyama, K, Todaka, D, et al. (2007). Functional analysis of a NAC-type transcription factor OsNAC6 involved in abiotic and biotic stress responsive gene expression in rice. Plant J., 51:617e30.

[48] Nakashima, K, Takasaki, H, Mizoi, J, Shinozaki, K, \& Yamaguchi-shinozaki, K. (2012). NAC transcription factors in plant abiotic stress responses. Biochimica et Biophysica Acta, , 1819, 97-103.

[49] Nakashima, K, Ito, Y, \& Yamaguchi-shinozaki, K. (2009). Transcriptional regulatory networks in response to abiotic stresses in Arabidopsis and grasses, Plant Physiol., , $149,88-95$.

[50] Nelson, D. E, Repetti, P. P, Adams, T. R, et al. (2007). Plant nuclear factor Y (NF-Y) B subunits confer drought tolerance and lead to improved corn yields on water-limited acres. Proc. Natl. Acad. Sci. U.S.A., , 104, 16450-16455.

[51] Ogata, K, \& Nishimura, Y. (1995). Specific DNA recognition by Myb protein. Tanpakushitsu Kakusan Koso, , 40, 1592-1597.

[52] Ogata, K. (1998). Structure and dynamics of the transcription factor, Myb, in DNAsequence recognition. Seikagaku, , 70, 1233-1250.

[53] Oh, S. J, Kim, Y. S, Kwon, C. W, Park, H. K, Jeong, J. S, \& Kim, J. K. (2009). Overexpression of the transcription factor AP37 in rice improves grain yield under drought conditions. Plant Physiol., , 150, 1368-1379.

[54] Paz-ares, J, Ghosal, D, Wienand, U, Peterson, P. A, \& Saedler, H. (1987). The regulatory c1 locus of Zea mays encodes a protein with homology to myb proto-oncogene products and with structural similarities to transcriptional activators. EMBO J., , 6, 3553-3558. 
[55] Peneli, L, Hallak-herr, E, Rozenberg, M, et al. (2002). Molecular and biochemical mechanisms associated with dormancy and drought tolerance in the desert legume Retama raetam. Plant J., , 31, 319-30.

[56] Proietti, S, Bertini, L, Van Der Ent, S, Leon-reyes, A, Pieterse, C. M. J, Tucci, M, Caporale, C, \& Caruso, C. (2011). Cross activity of orthologous WRKY transcription factors in wheat and Arabidopsis. J. Exp. Botany, , 62, 1975-1990.

[57] Qin, Y, Wang, M, Tian, Y, He, W, Han, L, \& Xia, G. (2012). Over-expression of TaMYB33 encoding a novel wheat MYB transcription factor increases salt and drought tolerance in Arabidopsis. Mol. Biol. Rep., , 39, 7183-7192.

[58] Rahaie, M, Xue, G-P, Naghavi, M. R, Alizadeh, H, \& Schenk, P. M. (2010). A MYB gene from wheat (Triticum aestivum L.) is up-regulated during salt and drought stresses and differentially regulated between salt-tolerant and sensitive genotypes. Plant Cell Rep., , 29, 835-844.

[59] Rahaie, M, Gomarian, M, Alizadeh, H, Malboobi, M. A, \& Naghavi, M. R. (2011). The expression analysis of transcription factors under long term salt stress in tolerant and susceptible wheat (Triticum aestivum L.) genotypes using Reverse Northern Blot. Iranian J. Crop Sci,

[60] Redillas, M. C. F. R, Jeong, J. S, Kim, Y. S, Jung, H, Bang, S. W, Choi, Y. D, Ha, S. H, Reuzeau, C, \& Kim, J. K. (2012). The overexpression of OsNAC9 alters the root architecture of rice plants enhancing drought resistance and grain yield under field conditions. Plant Biotechnol. J. , 10, 792-805.

[61] Rosinski, J. A, \& Atchley, W. R. (1998). Molecular evolution of the Myb family of transcription factors: evidence for polyphyletic origin. J. Mol. Evol., , 46, 74-83.

[62] Rushton, P. J, Macdonald, H, Huttly, A. K, et al. (1995). Members of a new family of DNA-binding proteins bind to a conserved cis-element in the promoters of a-Amy2 genes. Plant Mol, Biol., , 29, 691-702.

[63] Rushton, P. J, Torres, J. T, Parniske, M, et al. (1996). Interaction of elicitorinduced DNAbinding proteins with elicitor response elements in the promoters of parsley PR1 genes. EMBO J., , 15, 5690-700.

[64] Sakuma, Y, Maruyama, K, Osakabe, Y, Qin, F, Seki, M, Shinozaki, K, \& Yamaguchishinozaki, K. (2006). Functional analysis of an Arabidopsis transcription factor, DREB2A, involved in drought-responsive gene expression. Plant Cell, , 18, 1292-1309.

[65] Singh, K, Foley, R. C, \& Onate-sanchez, L. (2002). Transcription factors in plant defense and stress responses. Curr. Opin. Plant Biol., , 5, 430-436.

[66] Sun, C. X, Palmqvist, S, Olsson, H, et al. (2003). A Novel WRKY transcription factor, SUSIBA2, participates in sugar signaling in barley by binding to the sugar-responsive elements of the iso1 promoter. Plant Cell., , 15, 2076-92. 
[67] Takashi, K, Taga, Y, Takai, R, Iwano, M, Mastsui, H, Takayama, S, et al. (2009). The transcription factor OsNAC4 is a key positive regulator of plant hypersensitive cell death. EMBO J., 28:926e36.

[68] Tardif, G, Kane, N. A, \& Le, H. ne Adam, L. Labrie, G. Major, P. Gulick, F. Sarhan, J-F. Laliberte. (2007). Interaction network of proteins associated with abiotic stress response and development in wheat. Plant Mol. Biol., , 63, 703-718.

[69] Uno, Y, Furihata, T, Abe, H, Yoshida, R, Shinozaki, K, \& Yamaguchi-shinazachi, K. (2000). Arabidopsis basic leucine zipper transcription factors involved in an abscisic acid-dependent signal transduction pathways under drought and high-salinity conditions. Proc, Natl, Acad, Sci, USA, 97, 11632-11637.

[70] Velasco, R, Zharkikh, A, Troggio, M, et al. (2007). A high quality draft consensus sequence of the genome of a heterozygous grapevine variety. PLoS ONE 2, e1326.

[71] Wang, Z. P, Yang, P. Z, Fan, B. F, et al. (1998). An oligo selection procedure for identification of sequence-specific DNA-binding activities associated with the plant defense response. Plant J., , 16, 515-22.

[72] Wilkins, O, Nahal, H, Foong, J, Provart, N. J, \& Campbell, M. M. (2009). Expansion and diversification of the Populus R2R3-MYB family of transcription factors. Plant Physiol., , 149, 981-993.

[73] Wu, H, Ni, Z, Yao, Y, Guo, G, \& Sun, Q. (2008). Cloning and expression profiles of 15 genes encoding WRKY transcription factor in wheat (Triticum aestivem L.). Progress in Natural Science, , 18, 697-705.

[74] Xia, N, Zhang, G, Sun, Y-F, Zhu, L, Xu, L-S, Chen, X-M, Liu, B, Yu, Y-T, Wang, X-J, Huang, L-L, \& Kang, Z-S. (2010). TaNAC8, a novel NAC transcription factor gene in wheat, responds to stripe rust pathogen infection and abiotic stresses, Physiol. Mol. Plant Pathol., 74: 394e402.

[75] Xia, N, Zhang, G, Liu, X-Y, Deng, L, Cai, G-L, Zhang, Y, Wang, X-J, Zhao, J, Huang, LL, \& Kang, Z-S. (2010). Characterization of a novel wheat NAC transcription factor gene involved in defense response against stripe rust pathogen infection and abiotic stresses. Mol. Biol. Rep., , 37, 3703-3712.

[76] Xiao, B, Chen, Z, \& Xiang, X. C.-B., et al. (2009). Evaluation of seven function-known candidate genes for their effects on improving drought resistance of transgenic rice under field conditions. Mol. Plant, , 2, 73-83.

[77] Xu, Y. H, Wang, J. W, Wang, S, et al. (2004). Characterization of GaWRKY1, a cotton transcription factor that regulates the sesquiterpene synthase gene (+)- d-cadinene synthase-A. Plant Physiol., , 135, 507-15.

[78] Xue, G. P, Bower, N. I, Mcintyre, C. L, Riding, G. A, Kazan, K, \& Shorter, R. (2006). TaNAC69 from the NAC superfamily of transcription factors is up-regulated by abiotic stresses in wheat and recognizes two consensus DNA-binding sequences. Funct. Plant Biol., , 33, 43-57. 
[79] Xue, G. P, Way, H. M, Richardson, T, Drenth, J, Joyce, P. A, \& Mcintyre, C. L. (2011). Over-expression of TaNAC69 leads to enhanced transcript levels of stress up-regulated genes and dehydration tolerance in bread wheat. Mol. Plant, , 4, 697-712.

[80] Yamaguchi-shinozaki, K, Koizumi, M, Urao, S, \& Shinozaki, K. (1992). Molecular cloning and characterization of 9 cDNAs for genes that are responsive to desiccation in Arabidopsis thaliana: sequence analysis of one cDNA clone that encodes a putative transmembrane channel protein. Plant Cell Physiol., , 33, 217-224.

[81] Zhang, Y. J, \& Wang, L. J. (2005). The WRKY transcription factor superfamily: its origin in eukaryotes and expansion in plants. BMC Evol, Biol., 5:1.

[82] Zhang, L, Zhao, G, Xia, C, Jia, J, Liu, X, \& Kong, X. (2012). Overexpression of a wheat MYB transcription factor gene, TaMYB56-B, enhances tolerances to freezing and salt stresses in transgenic Arabidopsis. Gene, , 505, 100-107.

[83] Zhang, L, Zhao, G, Jia, J, Liu, X, \& Kong, X. (2012). Molecular characterization of 60 isolated wheat MYB genes and analysis of their expression during abiotic stress. J. Exp. Botany, , 63, 203-214.

[84] Zhang, L-C, Zhao, G-Y, Jia, J-Z, \& Kong, X-Y. (2009). Cloning and analysis of salt stressrelated gene TaMYB32 in wheat. Acta Agron. Sin., , 35, 1181-1187. 
